\title{
Individualised risk in patients undergoing lung volume reduction surgery: the Glenfield BFG score
}

\author{
Neil J. Greening ${ }^{1,2,5}$, Paul Vaughan ${ }^{3,5}$, Inger Oey ${ }^{3}$, Michael C. Steiner ${ }^{1,2,4}$, \\ Mike D. Morgan ${ }^{1}$, Sridhar Rathinam ${ }^{3}$ and David A. Waller ${ }^{3}$
}

Affiliations: ${ }^{1}$ Centre for Exercise and Rehabilitation Science, Institute of Lung Health, Leicester Respiratory Biomedical Research Unit, Dept of Respiratory Medicine, Glenfield Hospital, Leicester, UK. ${ }^{2}$ Dept of Infection, Inflammation and Immunity, University of Leicester, Leicester, UK. ${ }^{3}$ Dept of Thoracic Surgery, Glenfield Hospital, Leicester, UK. ${ }^{4}$ School of Sport, Exercise and Health Sciences, Loughborough University, Loughborough, UK. ${ }^{5}$ Authors contributed equally to work.

Correspondence: Neil J. Greening, Institute of Lung Health, Leicester Respiratory Biomedical Research Unit, Glenfield Hospital, Leicester, UK. Email: njg27ale.ac.uk

@ERSpublications

An individualised risk score for lung volume reduction surgery may aid decision making around surgery http://ow.ly/4lxm30b3n05

Cite this article as: Greening NJ, Vaughan P, Oey I, et al. Individualised risk in patients undergoing lung volume reduction surgery: the Glenfield BFG score. Eur Respir J 2017; 49: 1601766 [https://doi.org/ $10.1183 / 13993003.01766-2016]$.

ABSTRACT Lung volume reduction surgery (LVRS) has been shown to be beneficial in patients with chronic obstructive pulmonary disease, but there is low uptake, partly due to perceived concerns of high operative mortality. We aimed to develop an individualised risk score following LVRS.

This was a cohort study of patients undergoing LVRS. Factors independently predicting 90-day mortality and a risk prediction score were identified. Reliability of the score was tested using area under the receiver operating characteristic curve (AUROC).

237 LVRS procedures were performed. The multivariate analysis factors associated independently with death were: body mass index $(\mathrm{BMI})<18.5 \mathrm{~kg} \cdot \mathrm{m}^{-2}$ (OR 2.83, $\left.\mathrm{p}=0.059\right)$, forced expiratory volume in $1 \mathrm{~s}$ $\left(\mathrm{FEV}_{1}\right)<0.71 \mathrm{~L}(\mathrm{OR} 5.47, \mathrm{p}=0.011)$ and transfer factor of the lung for carbon monoxide $($ TLCO $)<20 \%(\mathrm{OR}$ 5.56, $\mathrm{p}=0.031$ ). A risk score was calculated and total score assigned. AUROC for the risk score was 0.80 and a better predictor than individual components $(\mathrm{p}<0.01)$. The score was stratified into three risk groups. Of the total patients, $46 \%$ were classified as low risk. Similar improvements in lung function and health status were seen in all groups. The score was introduced and tested in a further 71 patients. AUROC for 90 -day mortality in this cohort was 0.84 .

It is possible to provide an individualised predictive risk score for LVRS, which may aid decision making for both clinicians and patients.

Received: Sept 052016 | Accepted after revision: Feb 192017

Support statement: N.J. Greening is a National Institute for Health Research (NIHR) funded Clinical Lecturer. The study took place at the University Hospitals of Leicester NHS Trust. Support was also provided by the NIHR Leicester Respiratory Biomedical Research Unit. The views expressed are those of the authors and not necessarily those of the NHS, the NIHR or the Department of Health.

Conflict of interest: Disclosures can be found alongside this article at erj.ersjournals.com

Copyright OERS 2017 


\section{Introduction}

Chronic obstructive pulmonary disease (COPD) is a heterogeneous disease, with distinct disease patterns. In advanced disease, patients remain breathless despite proven therapies such as maximal bronchodilation and pulmonary rehabilitation. Lung volume reduction surgery (LVRS) has been shown to have maximum benefit in patients who are hyperinflated with heterogeneous emphysema, resulting in improvements in lung function, exercise capacity, health status and survival [1].

Despite the proven efficacy and cost-effectiveness [2] of LVRS, there remains a low rate of referral for assessment [3]. There are an estimated 16000 potentially eligible patients in the UK [4], yet only 89 VATS (video-assisted thoracoscopic surgery) LVRS procedures were performed in the UK in 2013-14 [5]. One of the major barriers to referral has been the perception by physicians of the high risk of the procedure [6], with a peri-operative mortality rate of $5.2 \%$ reported in those without high risk, and $7.9 \%$ in all patients [1]. However, individuals who undergo LVRS will differ in their peri-operative risk and there may be added value in the ability to present an objective, more individualised risk to a patient prior to surgical intervention. This may aid the multidisciplinary team (MDT) in decisions about eligibility for LVRS and alternative treatment strategies, as well as assist with shared decision making in the consultation with the patient. It is also unknown whether patients at differing peri-operative risk from LVRS confer a similar benefit in improvement, which may influence the risk-benefit balance of the intervention.

The purpose of this study was to identify pre-operative factors that are predictive of peri-operative mortality following LVRS. Using these risk factors we aimed to develop a scoring system to allow a better risk stratification that may aid patient selection, and the MDT discussion and decision, by individualising the peri-operative risk of such patients. We then aimed to assess the effectiveness of LVRS in each risk group and present its integration into clinical practice.

\section{Methods}

Study design

Prospectively collected data were obtained from a single-centre cohort of patients undergoing LVRS (Glenfield Hospital, Leicester, UK) as part of clinical practice. Patients with COPD were considered for LVRS if they were symptomatic with breathlessness despite optimised medical management, including pulmonary rehabilitation, were hyperinflated using body plethysmography (total lung capacity (TLC) $>100 \%$ predicted and either residual volume (RV) $>150 \%$ predicted or RV/TLC ratio $>55 \%$ ), and had evidence of emphysema heterogeneity - defined using quantitative radionuclide scintigraphy (Q scan) as a hypoperfused target area (contributing to $<10 \%$ of the perfusion to that lung). All patients who underwent LVRS were discussed at a dedicated MDT meeting as previously described, with a minimum of a respiratory physician, thoracic surgeon and radiologist in attendance [7]. Patients were routinely referred for pulmonary rehabilitation prior to the MDT discussion. A transfer factor of the lung for carbon monoxide (TLCO) $<20 \%$ was not considered an absolute contraindication to LVRS, and was considered if the forced respiratory volume in $1 \mathrm{~s}$ (FEV1) was $>20 \%$ predicted and written informed consent was obtained regarding the high risk of surgery. Only patients who underwent unilateral video-assisted thoracoscopy (VATS) were included in the analysis [8], including those undergoing second contralateral LVRS on a separate occasion to initial LVRS. No patients underwent single-stage bilateral LVRS during the analysis period. Contralateral LVRS was considered when deterioration was noted in patient perception of breathlessness and exercise capacity [9].

\section{Outcome measures}

The primary endpoint was mortality at 90 days. Mortality was captured from a dedicated prospective database, hospital records and GP records. All patients undergoing LVRS were followed up at a dedicated outpatient clinic every 3 months following surgery for 1 year. For those who died, date of death was recorded and post-operative interval calculated.

Demographics were collected at outpatient clinic prior to surgery. Lung function was assessed at Glenfield Hospital, and included assessment of static lung volumes estimated from body-box plethysmography and diffusion capacity. Lung function measures were obtained prior to MDT discussion, and at 3, 6 and 12 months following LVRS, at the time of the outpatient clinic review. Height and weight were measured at the outpatient clinic prior to surgery, and body mass index (BMI) calculated. Capillary blood gases were collected from arterialised ear lobes. Health status was measured using the EuroQol (EQ-5D) questionnaire at baseline and during follow-up clinic appointments or by postal survey.

\section{Statistical analysis}

All data were prospectively collected on a departmental database and retrospectively analysed. Statistical analysis was performed using Stata 13 (StataCorp, USA). Baseline characteristics between survivors and 
TABLE 1 Baseline pre-operative characteristics of survivors ( $n=216$ ) and patients who had died $(n=21)$ at 90 days post lung volume reduction surgery

\begin{tabular}{|c|c|c|c|c|}
\hline & Alive & Dead & Total & p-value \\
\hline Age years & $60 \pm 7$ & $62 \pm 8$ & $60 \pm 7$ & 0.310 \\
\hline Males & 61 & 67 & 61 & 0.589 \\
\hline$B M I \mathrm{~kg} \cdot \mathrm{m}^{-2}$ & $23.4 \pm 4.2$ & $21.8 \pm 5.3$ & $23.3 \pm 4.3$ & 0.110 \\
\hline MRC dyspnoea score & $4(3-4)$ & $4.5(3-5)$ & $4(3-4)$ & 0.478 \\
\hline Received long-term oxygen therapy & 13 & 17 & 13 & 0.643 \\
\hline Received ambulatory oxygen & 16 & 25 & 17 & \\
\hline $\mathrm{PaO}_{2} \mathrm{kPa}$ & $9.61 \pm 1.31$ & $9.39 \pm 1.42$ & $9.59 \pm 1.32$ & 0.484 \\
\hline $\mathrm{PaCO}_{2} \mathrm{kPa}$ & $5.28 \pm 0.65$ & $5.18 \pm 0.83$ & $5.27 \pm 0.83$ & 0.557 \\
\hline FEV 1 L & $0.81 \pm 0.34$ & $0.67 \pm 0.34$ & $0.79 \pm 0.34$ & 0.068 \\
\hline FEV $1 \%$ & $28.7 \pm 10.3$ & $23.5 \pm 5.9$ & $28.3 \pm 10.1$ & 0.024 \\
\hline FVC L & $2.53 \pm 0.86$ & $1.94 \pm 0.47$ & $2.48 \pm 0.85$ & 0.002 \\
\hline FVC $\%$ & $72.4 \pm 18.4$ & $63.0 \pm 20.2$ & $71.5 \pm 18.7$ & 0.028 \\
\hline TLC \% & $143 \pm 17$ & $139 \pm 16$ & $142 \pm 17$ & 0.326 \\
\hline RV \% & $261 \pm 54$ & $260 \pm 46$ & $261 \pm 53$ & 0.895 \\
\hline RV/TLC ratio \% & $66 \pm 9$ & $71 \pm 6$ & $67 \pm 9$ & 0.023 \\
\hline TLCO $\%$ & $39 \pm 13$ & $31 \pm 20$ & $38 \pm 14$ & 0.016 \\
\hline Kсо \% & $49 \pm 16$ & $42 \pm 33$ & $48 \pm 18$ & 0.146 \\
\hline
\end{tabular}

Data are presented as mean \pm SD, $\%$ and median (interquartile range). BMI: body mass index; MRC: Medical Research Council; $\mathrm{PaO}_{2}$ : partial pressure of arterial oxygen; $\mathrm{PaCO}_{2}$ : partial pressure of arterial carbon dioxide; FEV1: forced expiratory volume in $1 \mathrm{~s}$; FVC: forced vital capacity; TLC: total lung capacity; RV: residual volume; TLCO: transfer factor of the lung for carbon monoxide; KCO: transfer factor per unit alveolar volume.

patients who were dead at 90 days were compared using an independent sample t-test, Mann-Whitney U-test and Chi-squared test for parametric, non-parametric and categorical data respectively. Factors associated with 90 -day mortality were identified by univariate logistic regression. Multivariate analyses with the selected factors identified from the univariate analysis (selected using a cut-off p-value of 0.1 ) were conducted with binary logistic regression using a backward stepwise approach. Complete data for the multivariate model were available for 218 out of 237 (92\%) of the LVRS procedures. Continuous variables were grouped (FEV1, forced vital capacity (FVC), RV/TLC ratio divided at their mean;TLCO at cut-off based on the National Emphysema Treatment Trial (NETT) [1]; BMI at World Health Organization definition of underweight), allowing a risk score to be derived. To derive the risk score, a weighting was allocated to each risk factor based on the beta coefficient. Performance of the tool was assessed using receiver operating characteristic (ROC) curves. The area under the ROC curve (AUROC) was used to describe the overall performance of the score. Time to death was analysed using the Kaplain-Meier test. Differences between the three groups were compared using ANOVA.

The score derived from the first cohort was introduced to the LVRS MDT and was tested in a second cohort at the same site, on a separate occasion. Performance based on the derived score was tested using AUROC.

\section{Results}

A total of 237 unilateral VATS LVRS operations were performed over a 17-year period (1996-2013), excluding patients who underwent single-stage bilateral operations through VATS or sternotomy approaches. No difference in patient characteristics was seen over the study observation time period. Eight patients $(3.3 \%)$ who underwent LVRS had $a_{1}$-antitrypsin deficiency. LVRS was performed by two surgeons (SR, DAW) over this time period, with no difference in mortality between surgeons $(\mathrm{p}=0.315)$. Of the total LVRS procedures, 222 (94\%) were performed on the upper lobe, and 15 (6\%) on the lower lobe. Moreover, 32 patients (13.5\%) underwent contralateral LVRS at a mean interval of 3.4 years (SD 2.3 years) following the initial LVRS procedure. There were no differences in characteristics or outcomes between patients undergoing first and second procedures.

\section{Survival}

Overall mortality following LVRS was $4.2 \%(\mathrm{n}=10)$ at 30 days, $8.9 \%(\mathrm{n}=21)$ at 90 days and $12 \%(\mathrm{n}=28)$ at 1 year. Baseline demographics and clinical characteristics in patients who survived and died at 90 days are summarised in table 1. 
TABLE 2 Factors associated with death at 90 days in the univariate analysis

\begin{tabular}{|c|c|c|c|}
\hline & OR & $95 \% \mathrm{Cl}$ & p-value \\
\hline Age years & 1.04 & $0.97-1.11$ & 0.309 \\
\hline Males & 1.30 & $0.30-1.99$ & 0.590 \\
\hline $\mathrm{BMI}<18.5 \mathrm{~kg} \cdot \mathrm{m}^{-2}$ & 3.51 & $1.38-8.93$ & 0.008 \\
\hline Received long-term oxygen therapy & 1.58 & $0.30-8.19$ & 0.586 \\
\hline $\mathrm{PO}_{2} \mathrm{kPa}$ & 0.87 & $0.59-1.29$ & 0.481 \\
\hline $\mathrm{PCO}_{2} \mathrm{kPa}$ & 0.80 & $0.39-1.69$ & 0.555 \\
\hline FEV 1 L & 0.16 & $0.21-1.17$ & 0.071 \\
\hline FVC L & 0.33 & $0.16-0.68$ & 0.003 \\
\hline TLC L & 0.99 & $0.96-1.01$ & 0.325 \\
\hline RV \% pred & 0.99 & $0.99-1.01$ & 0.895 \\
\hline $\mathrm{RV} / \mathrm{TLC}$ ratio $\%$ pred & 1.07 & $1.01-1.13$ & 0.026 \\
\hline TLCO $(<20 \%)$ & 13.22 & $3.07-56.9$ & 0.001 \\
\hline TLCO $(20-39 \%)$ & 2.89 & $0.77-10.9$ & 0.116 \\
\hline
\end{tabular}

Significant variables are shown in bold. Odds ratios are per unit increase for continuous variables. For categorical variables, the group for which the odds ratio is shown is described. $\mathrm{BMI}$ : body mass index; $P_{\mathrm{O}_{2}}$ : partial pressure of oxygen; $P_{\mathrm{CO}_{2}}$ : partial pressure of carbon dioxide; $F E V 1$ : forced expiratory volume in $1 \mathrm{~s}$; FVC: forced vital capacity; TLC: total lung capacity; RV: residual volume; \% pred: \% predicted; TLCo: transfer factor of the lung for carbon monoxide.

Univariate logistic regression analyses for risk of death at 90 days identified a low BMI $\left(<18.5 \mathrm{~kg} \cdot \mathrm{m}^{-2}\right)$, $\mathrm{FEV}_{1}, \mathrm{FVC}, \mathrm{RV} / \mathrm{TLC}$ ratio and TLCO as significant predictors of death (table 2).

Multivariate analysis factors associated independently with death were: a BMI $<18.5 \mathrm{~kg} \cdot \mathrm{m}^{-2}$ (OR $2.83,95 \%$ CI 0.96-8.32, p=0.059), FEV1 <0.71 L (OR 5.47, 95\% CI 1.47-20.35, p=0.011) and TLCO <20\% (OR 5.56, 95\% CI 1.17-26.34, $\mathrm{p}=0.031)$. RV/TLC ratio and FVC did not contribute significantly to the model ( $\mathrm{p}=0.652$ and $\mathrm{p}=0.355$ respectively).

\section{Derivation of risk score}

Three independent factors were identified from the multivariate model: BMI "B", FEV1 "F" and gas transfer " $G$ ". Each factor was assigned a risk score based on its beta coefficient (nearest whole number) and then added together to produce a total score - the "Glenfield BFG" score. Table 3 describes the scoring system, with a range of $0-5$. The AUROC for the risk score and 90 -day mortality was 0.80 . The ROC curves for the individual components and the predictive score are shown in figure 1 . The risk score was a better predictor of outcome than any individual components of the score $(\mathrm{p}<0.01)$.

The predictive scores were divided into three groups: low risk (score 0-1), moderate risk (score 2-3) and high risk (score $4-5)$ groups; $46 \%(n=100), 41 \%(n=89)$ and $13 \%(n=29)$ of patients were classified in the low, moderate and high risk groups respectively. Kaplan-Meier survival, with separate curves for the three groups up to 1 year, is shown in figure 2. Significant differences in survival were seen between the three groups $(\mathrm{p}<0.01)$. Mortality rates at 30 days, 90 days and 1 year for the three groups are shown in table 4 .

\section{TABLE 3 Scoring system for risk of death post lung volume reduction surgery at 90 days}

\begin{tabular}{lcc} 
Variable & Measurement & Score \\
\hline BMI $\mathbf{~ k g} \cdot \mathbf{m}^{-2}$ & $>18.5$ & 0 \\
& $<18.5$ & 1 \\
TLCO $\%$ & $>40$ & 0 \\
& $20-39$ & 1 \\
FEV 1 L & $<20$ & 2 \\
& $>0.71$ & 0
\end{tabular}

Scores assigned and added together to attain total score: the Glenfield BFG score. Results based on pre-operative measures. BMI: body mass index; TLCO: transfer factor of the lung for carbon monoxide; FEV1: forced expiratory volume in $1 \mathrm{~s}$. 
FIGURE 1 Graph showing receiver operating characteristic (ROC) curve for the Glenfield BFG score and its individual components. Total area under the ROC curve (AUROC) for the Glenfield BFG score was 0.80 and a significantly better predictor of outcome $(p<0.01)$. TLCO: transfer factor of the lung for carbon monoxide; FEV1: forced expiratory volume in $1 \mathrm{~s}$; $\mathrm{BMI}$ : body mass index.

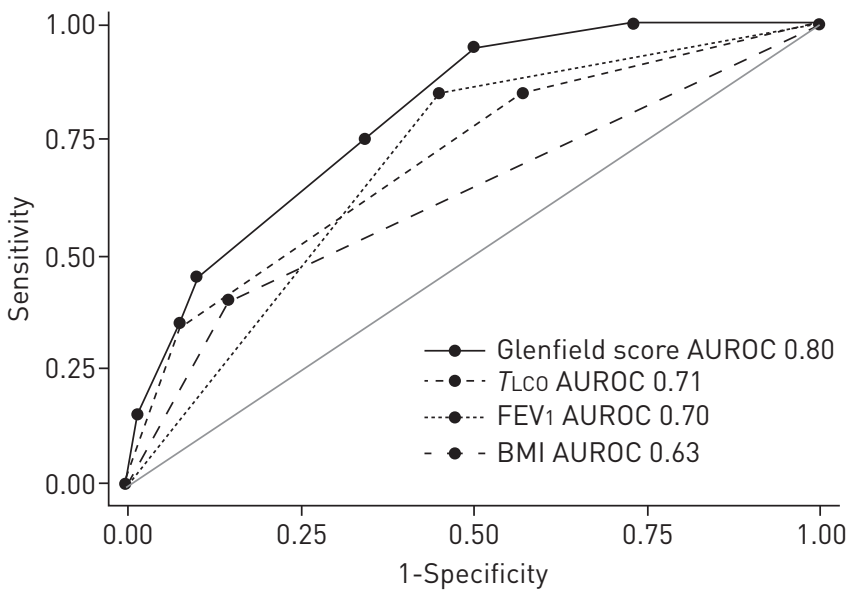

In addition to 90-day mortality, the Glenfield BFG score was tested at both shorter and longer time points. The AUROC for the different risk groups (low, moderate and high) at 30 days was 0.67 , and at 1 year was 0.80 .

\section{Clinical response of different risk groups}

Lung function was assessed at 3 months following LVRS. Significant increases in $\mathrm{FEV}_{1} \%$ predicted $(\mathrm{p}<0.05)$ and reduction in residual volume $(\mathrm{p}<0.01)$ were seen at 3 months following LVRS. There was no significant difference in lung function improvement in the different risk groups (figure 3 ).

Health status was measured using the EQ-5D. The proportion of patients with no symptoms, moderate symptoms and extreme symptoms in each risk group is shown in figure 4. Significant improvements were seen in symptoms of mobility $(\mathrm{p}<0.001)$, self-care $(\mathrm{p}=0.003)$ and activities $(\mathrm{p}=0.006)$, with a trend towards improvement in anxiety/depression $(\mathrm{p}=0.06)$. There were significantly worse symptoms of pain at 3 months $(p<0.001)$. No differences were found between the different risk groups in changes in health status ( $p>0.05$ for all EQ-5D domains).

\section{Testing of the Glenfield BFG risk score}

The Glenfield BFG risk score was introduced to the LVRS MDT in March 2013. Between March 2013 and July 2015, a further 71 patients underwent LVRS at Glenfield Hospital, Leicester, UK. This second cohort had a 90 -day mortality of $1.4 \%(n=1) ; 56 \%(n=39)$ were classified in the low-risk group, $40 \%(n=28)$ in the moderate-risk group and $4 \%(\mathrm{n}=3)$ in the high-risk group. The Glenfield score group remained a significant predictor of time to death (hazard ratio 18.8, 95\% CI 3.0-118.1, $\mathrm{p}=0.002$ ). At 90 days the Glenfield score had an AUROC of 0.84. A Kaplan-Meier survival curve of the three risk groups in the second cohort, following introduction of the Glenfield BFG score, is shown in figure 5.

\section{Discussion}

In this study we demonstrate that mortality following LVRS is acceptably low in selected groups, who can be identified from an individualised risk assessment score. Almost half (46\%) of our patients in this cohort

FIGURE 2 Kaplan-Meier survival of patients up to 1 year post lung volume reduction surgery (LVRS). Survival is shown based on the Glenfield BFG group. Low risk, score $0-1$; moderate risk, score 2-3; high risk, score 4-5.

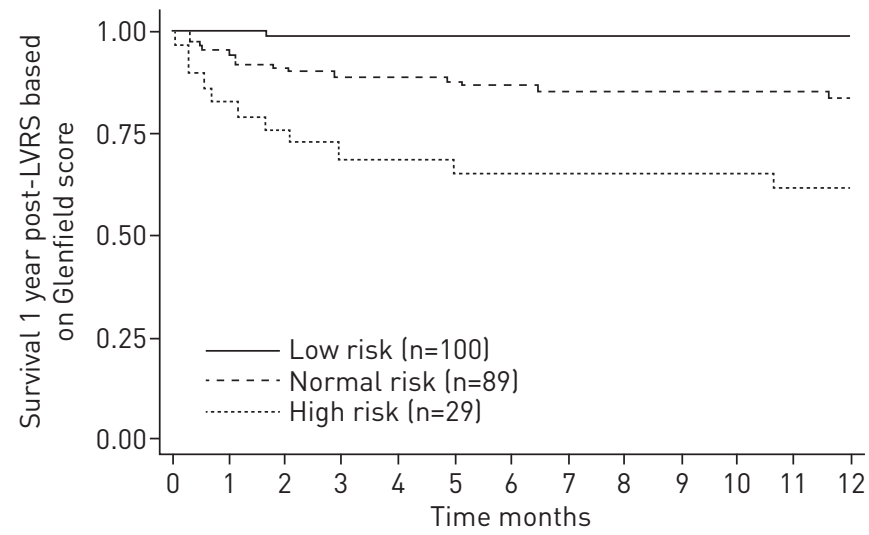


TABLE 4 Proportion of patients who died at 30 days, 90 days and 1 year in the three risk groups, as defined by the Glenfield BFG risk score

\begin{tabular}{lccc} 
& \multicolumn{2}{c}{ Mortality rate } \\
\cline { 2 - 4 } Glenfield score & $\mathbf{3 0}$ days & $\mathbf{9 0}$ days & $\mathbf{1}$ year \\
\hline Low 0-1 & 0.0 & 1.0 & 1.0 \\
Moderate 2-3 & 4.5 & 11.2 & 15.7 \\
High 4-5 & 17.2 & 31.0 & 37.9 \\
\hline
\end{tabular}

Data are presented as $\%$.

were classified in the lowest risk group. This population had only a $1 \%$ mortality at 90 days, which remained similar at 1 year. This study highlights that for many patients who undergo LRVS, it is of considerably lower risk than is often perceived [3]; this perception may deter many patients and referrers from considering LVRS.

We also show that routinely collected pre-operative measures can provide a more individualised risk assessment. This can be utilised using a simple scoring method - "the Glenfield BFG score" - which adds prognostic information. This multi-dimensional tool was superior to any of the individual factors for death. This is important as a patient may be considered high risk based on one measure, but have low risk for others, which influences overall risk.

All risk groups showed significant physiological benefit and improvements in health status following LVRS, with no inter-group difference. This suggests that whilst individuals may vary in their risk for LVRS, even high-risk patients may benefit from therapy. Whilst the Glenfield BFG score successfully identified patients at risk of death following LVRS, this does not measure factors that suggest benefit. Many of these have been previously identified in the NETT - for example, hyperinflation, disease heterogeneity and low exercise capacity.

We did see a significant worsening of symptoms in the pain domain of the EQ-5D post LVRS. This is not surprising and has been described previously [10], and is likely to improve as the time from surgery increases.

The Glenfield BFG score is practical to implement in the LVRS MDT, with the score successfully implemented in 2013, following its derivation. Fewer patients in the high-risk group underwent LVRS following implementation of the Glenfield BFG score (4\% versus 13\%) and were associated with a lower mortality rate compared with prior to introduction of the Glenfield BFG score. This would suggest it is a useful tool for the MDT and patients in informing decisions around risks of LVRS, though it is unknown whether this takes place during the MDT process or patient decision. However, patients at high risk still derived benefits in lung function and health status, similar to other groups, from LVRS and therefore careful discussion between clinicians and patients about the balance of potential risks and benefits requires careful consultation.

It is unsurprising that patients with the least severe disease markers have the lowest risk, and the factors identified are known risk factors for LVRS. A TLCO of $<20 \%$ predicted is considered a relative contraindication for surgery based on the NETT. This study agrees with previous work showing that a low
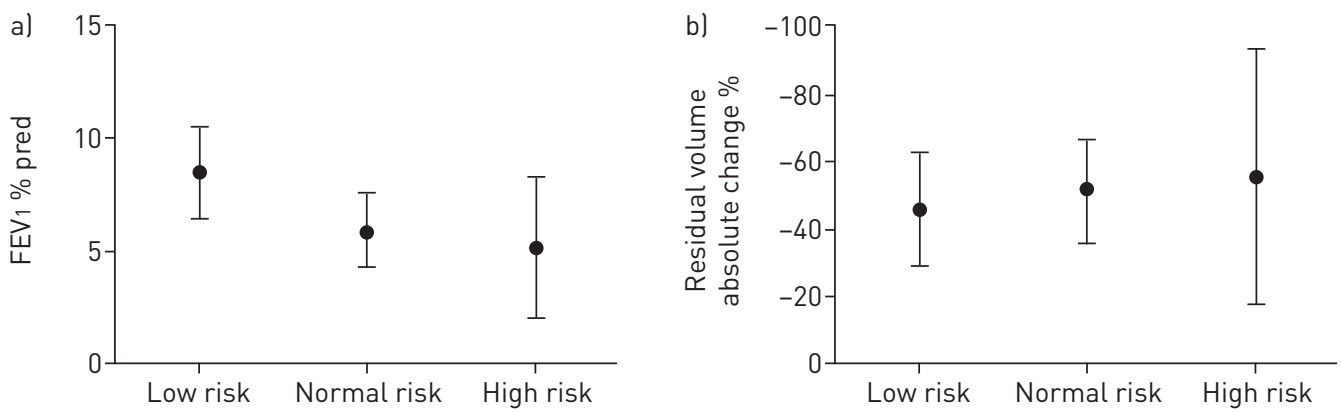

FIGURE 3 Lung function changes in patients alive at 90 days (mean \pm SD). Significant improvements in a) forced expiratory volume in $1 \mathrm{~s}\left(\mathrm{FEV}_{1}\right)$ and b) reduction in residual volume were seen in all groups. No difference was seen between groups. \% pred: \% predicted. 

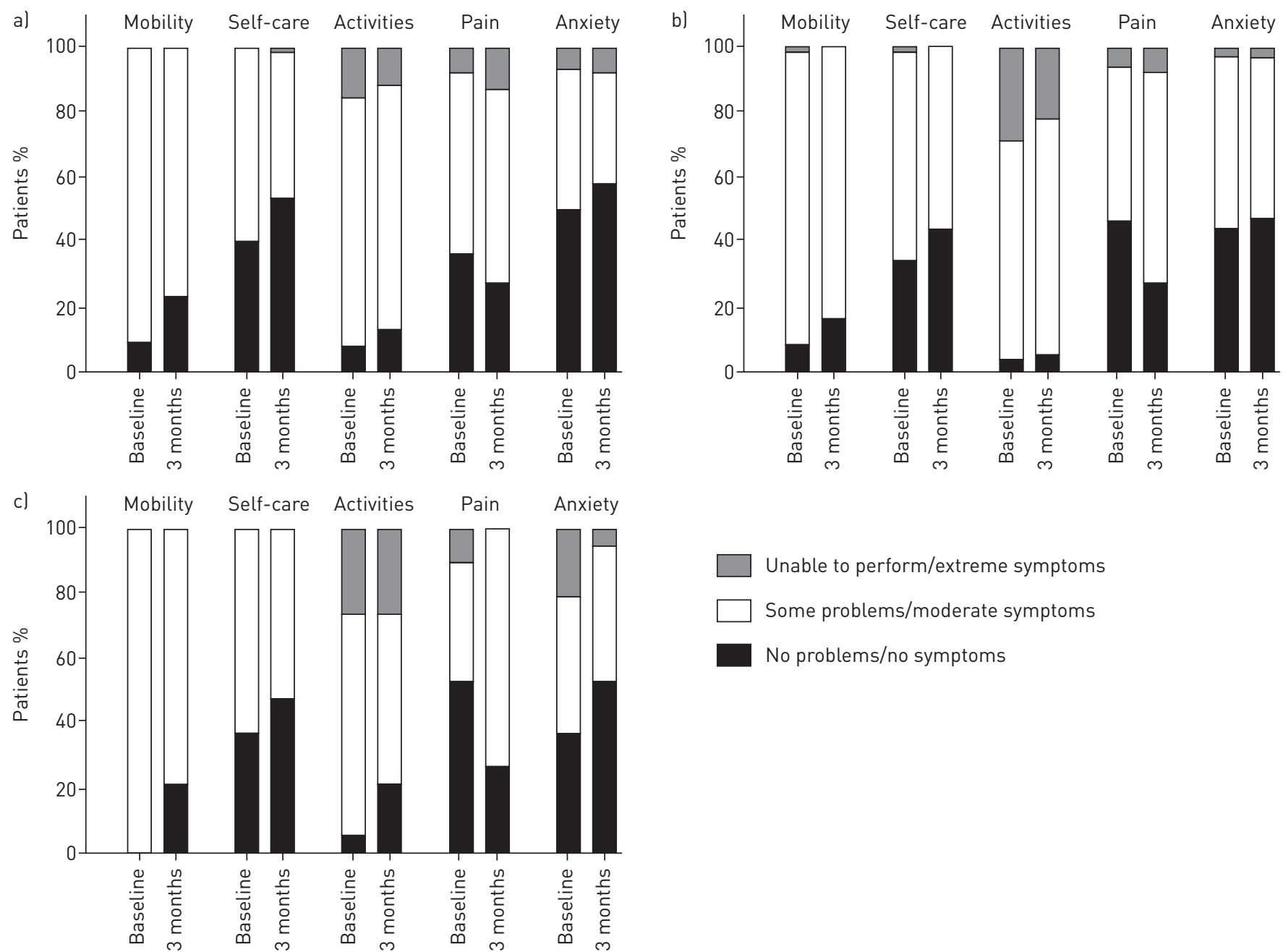

Unable to perform/extreme symptoms

Some problems/moderate symptoms

No problems/no symptoms

FIGURE 4 Health status changes in patients alive at 90 days. Significant improvements in EuroQol (EQ-5D) questionnaire mobility, self-care and activity subdomains were seen in all groups $(p<0.05)$. A trend to improvement in anxiety was seen $(p=0.06)$. There were significantly more symptoms in pain $(p<0.05)$. No difference was seen between groups: a) low-risk group; b) moderate-risk group; $c)$ high-risk group.

TLCO is an independent predictor of mortality, though this study would suggest that a TLCO $<20 \%$ predicted should be considered a relative contraindication depending on other risk factors. Our cut-off of $0.71 \mathrm{~L}$ for FEV1 is similar to the $20 \%$ predicted previously published high risk for LVRS [6]. The cut-off in our study was approximately $26 \%$ predicted. However, we used absolute values rather than predicted percentages. This is because calculation of predicted FEV1 requires height and weight values, and therefore interacts with BMI in the Glenfield BFG score, reducing its independence.

FIGURE 5 Kaplan-Meier survival of patients up to 1 year post lung volume reduction surgery (LVRS) in 71 patients following introduction of the Glenfield BFG score at the LVRS multidisciplinary team (MDT) discussion. Survival is shown based on the Glenfield BFG group. Low risk, score $0-1 ;$ moderate risk, score 2-3; high risk, score 4-5.

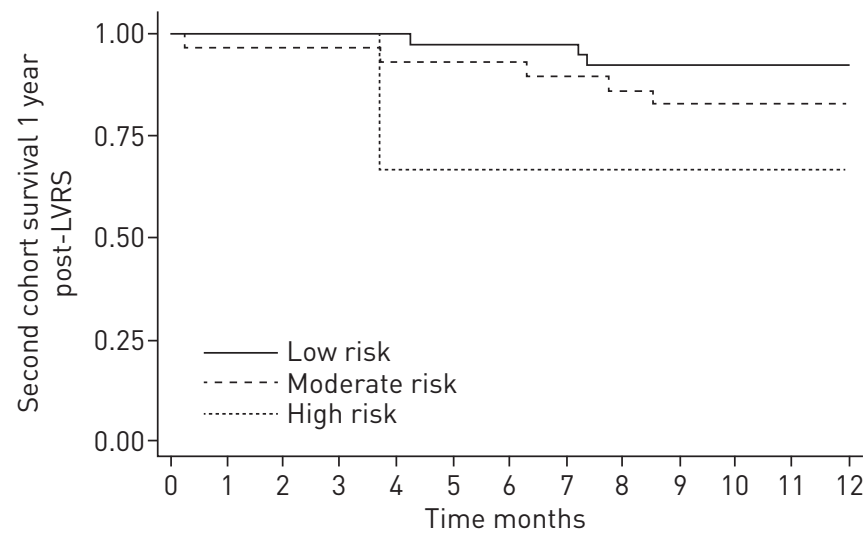


However, the Glenfield BFG score provides an objective and multi-dimensional system of scoring disease severity, superior to its individual elements. Given that patients with less severe markers of disease have a better prognosis and yet still gain significant benefit from LVRS, then earlier consideration of LVRS could be considered, especially if the potential risks are lower.

Similarly, identifying high-risk patients is necessary as it is important to counsel such patients appropriately in terms of the risks of the intervention, as other strategies may be more appropriate. This may include optimisation of symptom control through palliative care input, including alternative management of refractory dyspnoea or consideration of lung transplantation. However, this risk may be acceptable to patients; post-operative lung function and clinical outcome for high-risk patients are as good as for lower risk patients. Therefore, we believe that LVRS should still be considered, though a higher Glenfield BFG score may be considered a strong relative contraindication, and that patients should be counselled appropriately and be made aware of the risks involved. Those that were in the high-risk group typically had a low TLCO and a low FEV 1 . The high-risk group identified in this risk score had fulfilled the criteria for LVRS, including heterogeneous emphysema and absence of pulmonary hypertension.

A number of scoring systems and risk stratification tools exist for COPD. This is the first objective scoring system for risk of LVRS, with other scores typically focusing on long-term outcome [11] or exacerbation risk [12]. Other research has previously identified markers of high risk for LVRS, including homogenous disease, high perfusion ratio, low FEV1 $(<20 \%$ predicted) and low TLCO $(<20 \%$ predicted $)[1,6]$. These factors have led to changes in patient selection for LVRS. For example, all patients in this cohort had heterogeneous emphysema with low perfusion ratio, though in this cohort TLCO $<20 \%$ predicted was not considered an absolute contraindication.

The differences in mortality between the three risk groups were seen in the period following LVRS, with no separation in mortality after approximately 2 months (figure 2). This would suggest that the Glenfield BFG score is a true marker of peri-operative risk, rather than a marker of overall disease severity.

There are a number of potential mechanisms identified by the Glenfield BFG score that increase peri-operative adverse events. Malnutrition, measured by BMI in this study, leads to increased susceptibility to infection and poor wound healing. Specifically in COPD, poor nutritional status [11], frailty [13] and sarcopenia [14] are all associated with worse outcome. The Glenfield BFG score is also likely to identify patients at higher risk of prolonged air leak (PAL), as the higher risk patients had lower FEV1 and more severe emphysema, which are known risk factors for PAL [15]. PAL may then subsequently lead to increased morbidity and mortality $[16,17]$.

It is unknown whether the Glenfield score is applicable to newer endobronchial lung volume reduction (eLVR) techniques, such as endobronchial valves $[18,19]$ or coils $[20,21]$. The operative procedure in eLVR differs considerably from LVRS and may therefore alter peri-operative risk. However, the risk factors identified in the Glenfield BFG score may also be relevant for eLVR. Testing of the score following eLVR is therefore needed to validate it in this specific interventional population.

We acknowledge a number of limitations in this study. This is a retrospective cohort, although the data were collected prospectively. However, there will be bias within patient selection, though this has been standardised through a MDT and included high-risk patients. We would suggest that the role of the Glenfield BFG score would be to aid the MDT decision and allow patients to make a more informed judgement of risks. The data collection was conducted over a long period of time, and over time practice changes, which may alter mortality rate. However, LVRS is not a common procedure and contemporary cohorts of comparable size are not available. A lack of external contemporary cohorts means that we have not been able to provide external validation for the Glenfield BFG score, though prospective testing will be possible with new national LVR registries, such as those currently being established in the UK.

In summary, we have demonstrated and validated that using a simple scoring system, the "Glenfield BFG score", we can provide a more accurate peri-operative mortality risk for LVRS. The most frequent patient group was classified as low risk (mortality risk 1\%), which may increase LVRS uptake. Clinical benefit post-LVRS was seen in all risk groups and the score was successfully integrated into the LVR MDT. The Glenfield BFG score provides an individualised risk score for LVRS and may aid both clinician and patient in decision making around surgery.

\section{References}

1 Fishman A, Martinez F, Naunheim K, et al. A randomized trial comparing lung-volume-reduction surgery with medical therapy for severe emphysema. N Engl J Med 2003; 348: 2059-2073.

2 Ramsey SD, Berry K, Etzioni R, et al. Cost effectiveness of lung-volume-reduction surgery for patients with severe emphysema. N Engl J Med 2003; 348: 2092-2102. 
3 McNulty W, Jordan S, Hopkinson NS. Attitudes and access to lung volume reduction surgery for COPD: a survey by the British Thoracic Society. BMJ Open Respir Res 2014; 1: e000023.

4 Clark SJ, Zoumot Z, Bamsey O, et al. Surgical approaches for lung volume reduction in emphysema. Clin Med (Lond) 2014; 14: 122-127.

5 Society for Cardiothoracic Surgery in Great Britain \& Ireland. SCTS Thoracic Registry. www.scts.org/professionals/ audit_outcomes/thoracic.aspx Date last accessed: March 13, 2017.

6 National Emphysema Treatment Trial Research Group. Patients at high risk of death after lung-volume-reduction surgery. N Engl J Med 2001; 345: 1075-1083.

7 Rathinam S, Oey I, Steiner M, et al. The role of the emphysema multidisciplinary team in a successful lung volume reduction surgery programme. Eur J Cardiothorac Surg 2014; 46: 1021-1026.

8 Oey IF, Waller DA, Bal S, et al. Lung volume reduction surgery: a comparison of the long term outcome of unilateral vs. bilateral approaches. Eur J Cardiothorac Surg 2002; 22: 610-614.

9 Oey IF, Morgan MD, Spyt TJ, et al. Staged bilateral lung volume reduction surgery - the benefits of a patient-led strategy. Eur J Cardiothorac Surg 2010; 37: 846-852.

10 Oey IF, Morgan MD, Waller DA. Postoperative pain detracts from early health status improvement seen after video-assisted thoracoscopic lung volume reduction surgery. Eur J Cardiothorac Surg 2003; 24: 588-593.

11 Celli BR, Cote CG, Marin JM, et al. The body-mass index, airflow obstruction, dyspnea, and exercise capacity index in chronic obstructive pulmonary disease. N Engl J Med 2004; 350: 1005-1012.

12 Jones RC, Donaldson GC, Chavannes NH, et al. Derivation and validation of a composite index of severity in chronic obstructive pulmonary disease: the DOSE Index. Am J Respir Crit Care Med 2009; 180: 1189-1195.

13 Kon SS, Jones SE, Schofield SJ, et al. Gait speed and readmission following hospitalisation for acute exacerbations of COPD: a prospective study. Thorax 2015; 70: 1131-1137.

14 Greening NJ, Harvey-Dunstan TC, Chaplin EJ, et al. Bedside assessment of quadriceps muscle by ultrasound after admission for acute exacerbations of chronic respiratory disease. Am J Respir Crit Care Med 2015; 192: 810-816.

15 Elsayed H, McShane J, Shackcloth M. Air leaks following pulmonary resection for lung cancer: is it a patient or surgeon related problem? Ann R Coll Surg Engl 2012; 94: 422-427.

16 Mueller MR, Marzluf BA. The anticipation and management of air leaks and residual spaces post lung resection. $J$ Thorac Dis 2014; 6: 271-284.

17 DeCamp MM, Blackstone EH, Naunheim KS, et al. Patient and surgical factors influencing air leak after lung volume reduction surgery: lessons learned from the National Emphysema Treatment Trial. Ann Thorac Surg 2006; 82: 197-206.

18 Klooster K, ten Hacken NH, Hartman JE, et al. Endobronchial valves for emphysema without interlobar collateral ventilation. N Engl J Med 2015; 373: 2325-2335.

19 Davey C, Zoumot Z, Jordan S, et al. Bronchoscopic lung volume reduction with endobronchial valves for patients with heterogeneous emphysema and intact interlobar fissures (the BeLieVeR-HIFi study): a randomised controlled trial. Lancet 2015; 386: 1066-1073.

20 Deslee G, Klooster K, Hetzel M, et al. Lung volume reduction coil treatment for patients with severe emphysema: a European multicentre trial. Thorax 2014; 69: 980-986.

21 Shah PL, Zoumot Z, Singh S, et al. Endobronchial coils for the treatment of severe emphysema with hyperinflation (RESET): a randomised controlled trial. Lancet Respir Med 2013; 1: 233-240. 